\title{
Observer-Based Robust Control of Uncertain Fuzzy Models with Pole Placement Constraints
}

\author{
Pagès Olivier and El Hajjaji Ahmed \\ University of Picardie Jules Verne, MIS, Amiens
}

France

\section{Introduction}

Practical systems are often modelled by nonlinear dynamics. Controlling nonlinear systems are still open problems due to their complexity nature. This problem becomes more complex when the system parameters are uncertain. To control such systems, we may use the linearization technique around a given operating point and then employ the known methods of linear control theory. This approach is successful when the operating point of the system is restricted to a certain region. Unfortunately, in practice this approach will not work for some physical systems with a time-varying operating point. The fuzzy model proposed by Takagi-Sugeno (T-S) is an alternative that can be used in this case. It has been proved that T-S fuzzy models can effectively approximate any continuous nonlinear systems by a set of local linear dynamics with their linguistic description. This fuzzy dynamic model is a convex combination of several linear models. It is described by fuzzy rules of the type If-Then that represent local input output models for a nonlinear system. The overall system model is obtained by "blending" these linear models through nonlinear fuzzy membership functions. For more details on this topic, we refer the reader to (Tanaka \& al 1998 and Wand \& al, 1995) and the references therein.

The stability analysis and the synthesis of controllers and observers for nonlinear systems described by T-S fuzzy models have been the subject of many research works in recent years. The fuzzy controller is often designed under the well-known procedure: Parallel Distributed Compensation (PDC). In presence of parametric uncertainties in T-S fuzzy models, it is necessary to consider the robust stability in order to guarantee both the stability and the robustness with respect to the latter. These may include modelling error, parameter perturbations, external disturbances, and fuzzy approximation errors. So far, there have been some attempts in the area of uncertain nonlinear systems based on the T-S fuzzy models in the literature. The most of these existing works assume that all the system states are measured. However, in many control systems and real applications, these are not always available. Several authors have recently proposed observer based robust controller design methods considering the fact that in real control problems the full state information is not always available. In the case without uncertainties, we apply the separation property to design the observer-based controller: the observer synthesis is designed so that its dynamics are fast and we independently design the controller by imposing slower dynamics. Recently, much effort has been devoted to observer-based control for T-S fuzzy models. (Tanaka \& al, 1998) have studied the fuzzy observer design for T-S fuzzy control systems. Nonetheless, in 
the presence of uncertainties, the separation property is not applicable any more. In (El Messousi \& al, 2006), the authors have proposed sufficient global stability conditions for the stabilization of uncertain fuzzy T-S models with unavailable states using a robust fuzzy observer-based controller but with no consideration to the control performances and in particular to the transient behaviour.

From a practical viewpoint, it is necessary to find a controller which will specify the desired performances of the controlled system. For example, a fast decay, a good damping can be imposed by placing the closed-loop poles in a suitable region of the complex plane. Chilali and Gahinet (Chilali \& Gahinet, 1996) have proposed the concept of an LMI (Linear Matrix Inequality) region as a convenient LMI-based representation of general stability regions for uncertain linear systems. Regions of interest include a-stability regions, disks and conic sectors. In (Chilali \& al 1999), a robust pole placement has been studied in the case of linear systems with static uncertainties on the state matrix. A vertical strip and a-stability robust pole placement has been studied in (Wang \& al, 1995, Wang \& al, 1998 and Wang \& al, 2001) respectively for uncertain linear systems in which the concerned uncertainties are polytopic and the proposed conditions are not LMI. In (Hong \& Man 2003), the control law synthesis with a pole placement in a circular LMI region is presented for certain T-S fuzzy models. Different LMI regions are considered in (Farinwata \& al, 2000 and Kang \& al, 198), for closed-loop pole placements in the case of T-S fuzzy models without uncertainties.

In this work, we extend the results of (El Messoussi \& al, 2005), in which we have developed sufficient robust pole placement conditions for continuous T-S fuzzy models with measurable state variables and structured parametric uncertainties.

The main goal of this paper is to study the pole placement constraints for T-S fuzzy models with structured uncertainties by designing an observer-based fuzzy controller in order to guarantee the closed-loop stability. However, like (Lo \& Li, 2004 and Tong \& Li, 2002), we do not know the position of the system state poles as well as the position of the estimation error poles. The main contribution of this paper is as follows: the idea is to place the poles associated with the state dynamics in one LMI region and to place the poles associated with the estimation error dynamics in another LMI region (if possible, farther on the left). However, the separation property is not applicable unfortunately. Moreover, the estimation error dynamics depend on the state because of uncertainties. If the state dynamics are slow, we will have a slow convergence of the estimation error to the equilibrium point zero in spite of its own fast dynamics. So, in this paper, we propose an algorithm to design the fuzzy controller and the fuzzy observer separately by imposing the two pole placements. Moreover, by using the $H_{\infty}$ approach, we ensure that the estimation error converges faster to the equilibrium point zero.

This chapter is organized as follows: in Section 2, we give the class of uncertain fuzzy models, the observer-based fuzzy controller structure and the control objectives. After reviewing existing LMI constraints for a pole placement in Section 3, we propose the new conditions for the uncertain augmented T-S fuzzy system containing both the fuzzy controller as well as the observer dynamics. Finally, in Section 4, an illustrative application example shows the effectiveness of the proposed robust pole placement approach. Some conclusions are given in Section 5.

\section{Problem formulation and preliminaries}

Considering a T-S fuzzy model with parametric uncertainties composed of $r$ plant rules that can be represented by the following fuzzy rule: 


\section{Plant rule $i$ :}

If $z_{1}(t)$ is $M_{1 i}$ and $\ldots$ and $z_{v}(t)$ is $M_{v i}$ Then $\left\{\begin{array}{l}\dot{x}(t)=\left(A_{i}+\Delta A_{i}\right) x(t)+\left(B_{i}+\Delta B_{i}\right) u(t), \\ y(t)=C_{i} x(t) \quad i=1, \ldots, r\end{array}\right.$

The structured uncertainties considered here are norm-bounded in the form:

$$
\begin{aligned}
\Delta A_{i} & =H_{a i} \Delta_{a i}(t) E_{a i}, \\
\Delta B_{i} & =H_{b i} \Delta_{b i}(t) E_{b i}, i=1, \ldots, r
\end{aligned}
$$

Where $H_{a i}, H_{b i}, E_{a i}, E_{b i}$ are known real constant matrices of appropriate dimension, and $\Delta_{a i}(t), \Delta_{b i}(t)$ are unknown matrix functions satisfying:

$$
\begin{aligned}
& \Delta_{a i}^{t}(t) \Delta_{a i}(t) \leq I, \\
& \Delta_{b i}^{t}(t) \Delta_{b i}(t) \leq I \quad i=1, \ldots, r
\end{aligned}
$$

$\Delta_{a i}^{t}(t)$ is the transposed matrix of $\Delta_{a i}(t)$ and $I$ is the matrix identity of appropriate dimension. We suppose that pairs $\left(A_{i}, B_{i}\right)$ are controllable and $\left(A_{i}, C_{i}\right)$ are observable. $M_{i j}$ indicates the $j^{\text {th }}$ fuzzy set associated to the $i^{\text {th }}$ variable $z_{i}(t), r$ is the number of fuzzy model rules, $x(t) \in \mathfrak{R}^{n}$ is the state vector, $u(t) \in \mathfrak{R}^{m}$ is the input vector, $y(t) \in R^{l}$ is the output vector, $A_{i} \in \mathfrak{R}^{n \times n}, B_{i} \in \mathfrak{R}^{n \times m}$ and $C_{i} \in \mathfrak{R}^{l \times n} . z_{1}(t), \ldots, z_{v}(t)$ are premise variables.

From (1), the T-S fuzzy system output is :

$$
\left\{\begin{array}{l}
\dot{x}(t)=\sum_{i=1}^{r} h_{i}(z(t))\left[\left(A_{i}+\Delta A_{i}\right) x(t)+\left(B_{i}+\Delta B_{i}\right) u(t)\right] \\
y(t)=\sum_{i=1}^{r} h_{i}(z(t)) C_{i} x(t)
\end{array}\right.
$$

where $\quad h_{i}(z(t))=\frac{w_{i}(z(t))}{\sum_{i=1}^{r} w_{i}(z(t))}$ and $w_{i}(z(t))=\prod_{j=1}^{v} \mu_{M_{i j}}\left(z_{j}(t)\right)$

Where $\mu_{M_{i j}}\left(z_{j}(t)\right)$ is the fuzzy meaning of symbol $M_{i j}$.

In this paper we assume that all of the state variables are not measurable. Fuzzy state observer for T-S fuzzy model with parametric uncertainties (1) is formulated as follows: Observer rule $i$ :

$$
\text { If } z_{1}(t) \text { is } M_{1 i} \text { and } \ldots \text { and } z_{v}(t) \text { is } M_{v i} \text { Then }\left\{\begin{array}{l}
\dot{\hat{x}}(t)=A_{i} \hat{x}(t)+B_{i} u(t)-G_{i}(y(t)-\hat{y}(t)) \text {, } \\
\hat{y}(t)=C_{i} \hat{x}(t) \quad i=1, \ldots, r
\end{array}\right.
$$

The fuzzy observer design is to determine the local gains $G_{i} \in \mathfrak{R}^{n \times l}$ in the consequent part. Note that the premise variables do not depend on the state variables estimated by a fuzzy observer.

The output of (5) is represented as follows: 


$$
\left\{\begin{array}{l}
\dot{\hat{x}}(t)=\sum_{i=1}^{r} h_{i}(z(t))\left\{A_{i} \hat{x}(t)+B_{i} u(t)-G_{i}(y(t)-\hat{y}(t))\right\} \\
\hat{y}(t)=\sum_{i=1}^{r} h_{i}(z(t)) C_{i} \hat{x}(t)
\end{array}\right.
$$

To stabilize this class of systems, we use the PDC observer-based approach (Tanaka \& al, 1998). The PDC observer-based controller is defined by the following rule base system:

Controller rule $i$ :

$$
\text { If } z_{1}(t) \text { is } M_{1 i} \text { and } \ldots \text { and } z_{v}(t) \text { is } M_{v i} \text { Then } u(t)=K_{i} \hat{x}(t) \quad i=1, \ldots, r
$$

The overall fuzzy controller is represented by:

$$
u(t)=\frac{\sum_{i=1}^{r} w_{i}(z(t)) K_{i} \hat{x}(t)}{\sum_{i=1}^{r} w_{i}(z(t))}=\sum_{i=1}^{r} h_{i}(z(t)) K_{i} \hat{x}(t)
$$

Let us denote the estimation error as:

$$
e(t)=x(t)-\hat{x}(t)
$$

The augmented system containing both the fuzzy controller and observer is represented as follows:

$$
\left[\begin{array}{l}
\dot{x}(t) \\
\dot{e}(t)
\end{array}\right]=\bar{A}(z(t)) \times\left[\begin{array}{l}
x(t) \\
e(t)
\end{array}\right]
$$

where

$$
\begin{aligned}
& \bar{A}(z(t))=\sum_{i=1}^{r} \sum_{j=1}^{r} h_{i}(z(t)) h_{j}(z(t)) \bar{A}_{i j} \\
& \bar{A}_{i j}=\left[\begin{array}{cc}
\left(A_{i}+\Delta A_{i}\right)+\left(B_{i}+\Delta B_{i}\right) K_{j} & -\left(B_{i}+\Delta B_{i}\right) K_{j} \\
\left(\Delta A_{i}+\Delta B_{i} K_{j}\right) & \left(A_{i}+G_{i} C_{j}-\Delta B_{i} K_{j}\right)
\end{array}\right]
\end{aligned}
$$

The main goal is first, to find the sets of matrices $K_{i}$ and $G_{i}$ in order to guarantee the global asymptotic stability of the equilibrium point zero of (10) and secondly, to design the fuzzy controller and the fuzzy observer of the augmented system (10) separately by assigning both "observer and controller poles" in a desired region in order to guarantee that the error between the state and its estimation converges faster to zero. The faster the estimation error will converge to zero, the better the transient behaviour of the controlled system will be.

\section{Main results}

Given (1), we give sufficient conditions in order to satisfy the global asymptotic stability of the closed-loop for the augmented system (10). 
Lemma 1: The equilibrium point zero of the augmented system described by (10) is globally asymptotically stable if there exist common positive definite matrices $P_{1}$ and $P_{2}$, matrices $W_{i}, V_{j}$ and positive scalars $\varepsilon_{i j} \succ 0$ such as

$$
\begin{aligned}
& \Pi_{i i} \leq 0, \quad i=1, \ldots, r \\
& \Pi_{i j}+\Pi_{j i} \leq 0, i<j \leq r
\end{aligned}
$$

And

$$
\begin{aligned}
& \Sigma_{i i} \leq 0, \quad i=1, \ldots, r \\
& \Sigma_{i j}+\Sigma_{j i} \leq 0, \quad i<j \leq r
\end{aligned}
$$

with

$$
\begin{aligned}
\Pi_{i j}= & {\left[\begin{array}{ccccc}
D_{i j} & P_{1} E_{a i}^{t} & V_{j}^{t} E_{b i}^{t} & B_{i} & H_{b i} \\
E_{a i} P_{1} & -0.5 \varepsilon_{i j} I & 0 & 0 & 0 \\
E_{b i} V_{j} & 0 & -0.5 \varepsilon_{i j} I & 0 & 0 \\
B_{i}^{t} & 0 & 0 & -\varepsilon_{i j} I & 0 \\
H_{b i}^{t} & 0 & 0 & 0 & -\varepsilon_{i j} I
\end{array}\right] \sum_{i j}=\left[\begin{array}{ccccc}
D_{i j}^{*} & K_{j}^{t} E_{b i}^{t} & P_{2} H_{a i} & P_{2} H_{b i} & K_{j}^{t} \\
E_{b i} K_{j} & -\varepsilon_{i j}^{-1} I & 0 & 0 & 0 \\
H_{a i}^{t} P_{2} & 0 & -\varepsilon_{i j}^{-1} I & 0 & 0 \\
H_{b i}^{t} P_{2} & 0 & 0 & -0.5 \varepsilon_{i j}^{-1} I & 0 \\
K_{j} & 0 & 0 & 0 & -\varepsilon_{i j}^{-1} I
\end{array}\right] } \\
D_{i j} & =A_{i} P_{1}+P_{1} A_{i}^{t}+B_{i} V_{j}+V_{j}^{t} B_{i}^{t}+\varepsilon_{i j} H_{a i} H_{a i}^{t}+\varepsilon_{i j} H_{b i} H_{b i}^{t} \\
D_{i j}^{*} & =P_{2} A_{i}+A_{i}^{t} P_{2}+W_{i} C_{j}+C_{j}^{t} W_{i}^{t}+\varepsilon_{i j}^{-1} K_{j}^{t} E_{b i}^{t} E_{b i} K_{j}
\end{aligned}
$$

Proof: using theorem 7 in (Tanaka \& al, 1998), property (3), the separation lemma (Shi \& al, 1992)) and the Schur's complement (Boyd \& al, 1994), the above conditions (12) and (13) hold with some changes of variables. Let us briefly explain the different steps...

From (11), in order to ensure the global, asymptotic stability, the sufficient conditions must be verified:

$$
\exists X=X^{t}>0: M_{D}(\bar{A}, X)=\bar{A}_{i j} X+X \bar{A}_{i j}^{t}<0
$$

Let: $X=\left[\begin{array}{cc}X_{11} & 0 \\ 0 & X_{22}\end{array}\right]$ where 0 is a zero matrix of appropriate dimension. From (14), we have:

$$
M_{D}(\bar{A}, X)=M_{D}^{1}+M_{D}^{2}
$$

With $M_{D}^{1}=\left[\begin{array}{cc}D_{1} & 0 \\ 0 & D_{2}\end{array}\right]$ where

$$
D_{1}=A_{i} X_{11}+X_{11} A_{i}^{t}+B_{i} K_{j} X_{11}+X_{11} K_{j}^{t} B_{i}^{t}
$$

and

$$
D_{2}=A_{i} X_{22}+X_{22} A_{i}^{t}+G_{i} C_{j} X_{22}+X_{22} C_{j}^{t} G_{i}^{t}
$$


From (15),

$$
M_{D}^{2}=\left[\begin{array}{cc}
\Delta_{1} & X_{11} \Delta A_{i}^{t}+X_{11} K_{j}^{t} \Delta B_{i}^{t}-B_{i} K_{j} X_{22}-\Delta B_{i} K_{j} X_{22} \\
\Delta A_{i} X_{11}+\Delta B_{i} K_{j} X_{11}-X_{22} K_{j}^{t} B_{i}^{t}-X_{22} K_{j}^{t} \Delta B_{i}^{t} & \Delta_{2}
\end{array}\right]
$$

where $\Delta_{1}=\Delta A_{i} X_{11}+X_{11} \Delta A_{i}^{t}+\Delta B_{i} K_{j} X_{11}+X_{11} K_{j}^{t} \Delta B_{i}^{t}$ and $\Delta_{2}=-\Delta B_{i} K_{j} X_{22}-X_{22} K_{j}^{t} \Delta B_{i}^{t}$

From (15), we have:

$M_{D}^{2}=\Sigma_{1}+\Sigma_{2}+\Sigma_{3}$ with $\Sigma_{1}=\left[\begin{array}{cc}0 & -B_{i} K_{j} X_{22}-\Delta B_{i} K_{j} X_{22} \\ -X_{22} K_{j}^{t} B_{i}^{t}-X_{22} K_{j}^{t} \Delta B_{i}^{t} & 0\end{array}\right]$,

$\Sigma_{2}=\left[\begin{array}{cc}0 & X_{11} \Delta A_{i}^{t}+X_{11} K_{j}^{t} \Delta B_{i}^{t} \\ \Delta A_{i} X_{11}+\Delta B_{i} K_{j} X_{11} & 0\end{array}\right]$ and $\Sigma_{3}=\left[\begin{array}{cc}\Delta_{1} & 0 \\ 0 & \Delta_{2}\end{array}\right]$

Let $X_{11}=P_{1}, X_{11}=P_{2}^{-1}$. From the previous equation and (2), we have:

$$
\begin{aligned}
& \Sigma_{1}=\left[\begin{array}{cc}
0 & 0 \\
0 & -P_{2}^{-1} K_{j}^{t}
\end{array}\right] \times\left[\begin{array}{cc}
0 & 0 \\
B_{i}^{t} & 0
\end{array}\right]+\left[\begin{array}{cc}
0 & B_{i} \\
0 & 0
\end{array}\right] \times\left[\begin{array}{cc}
0 & 0 \\
0 & -K_{j} P_{2}^{-1}
\end{array}\right]+\left[\begin{array}{cc}
0 & 0 \\
0 & -P_{2}^{-1} K_{j}^{t} E_{b i}^{t}
\end{array}\right] \times\left[\begin{array}{cc}
0 & 0 \\
\Delta_{b i}^{t} H_{b i}^{t} & 0
\end{array}\right] \\
& +\left[\begin{array}{cc}
0 & H_{b i} \Delta_{b i} \\
0 & 0
\end{array}\right] \times\left[\begin{array}{cc}
0 & 0 \\
0 & -E_{b i} K_{j} P_{2}^{-1}
\end{array}\right]
\end{aligned}
$$

And,

$$
\begin{aligned}
& \Sigma_{2}=\left[\begin{array}{cc}
0 & 0 \\
H_{a i} \Delta_{a i} & 0
\end{array}\right] \times\left[\begin{array}{cc}
E_{a i} P_{1} & 0 \\
0 & 0
\end{array}\right]+\left[\begin{array}{cc}
P_{1} E_{a i}^{t} & 0 \\
0 & 0
\end{array}\right] \times\left[\begin{array}{cc}
0 & \Delta_{a i}^{t} H_{a i}^{t} \\
0 & 0
\end{array}\right]+\left[\begin{array}{cc}
0 & 0 \\
H_{b i} \Delta_{b i} & 0
\end{array}\right] \times\left[\begin{array}{cc}
E_{b i} K_{j} P_{1} & 0 \\
0 & 0
\end{array}\right] \\
& +\left[\begin{array}{cc}
P_{1} K_{j}^{t} E_{b i}^{t} & 0 \\
0 & 0
\end{array}\right] \times\left[\begin{array}{cc}
0 & \Delta_{b i}^{t} H_{b i}^{t} \\
0 & 0
\end{array}\right]
\end{aligned}
$$

And finally:

$$
\begin{aligned}
& \Sigma_{3}=\left[\begin{array}{cc}
H_{a i} \Delta_{a i} & H_{b i} \Delta_{b i} \\
0 & 0
\end{array}\right] \times\left[\begin{array}{cc}
E_{a i} P_{1} & 0 \\
E_{b i} K_{j} P_{1} & 0
\end{array}\right]+\left[\begin{array}{cc}
P_{1} E_{a i}^{t} & P_{1} K_{j}^{t} E_{b i}^{t} \\
0 & 0
\end{array}\right] \times\left[\begin{array}{cc}
\Delta_{a i}^{t} H_{a i}^{t} & 0 \\
\Delta_{b i}^{t} H_{b i}^{t} & 0
\end{array}\right] \\
& +\left[\begin{array}{cc}
0 & 0 \\
0 & -H_{b i} \Delta_{b i}
\end{array}\right] \times\left[\begin{array}{cc}
0 & 0 \\
0 & E_{b i} K_{j} P_{2}^{-1}
\end{array}\right]+\left[\begin{array}{cc}
0 & 0 \\
0 & P_{2}^{-1} K_{j}^{t} E_{b i}^{t}
\end{array}\right] \times\left[\begin{array}{cc}
0 & 0 \\
0 & -\Delta_{b i}^{t} H_{b i}^{t}
\end{array}\right]
\end{aligned}
$$

From (18), (19) and (20) and by using the separation lemma (Shi \& al, 1992)), we finally obtain:

Where:

$$
M_{D}^{2} \leq\left[\begin{array}{cc}
T_{1} & 0 \\
0 & T_{2}
\end{array}\right]
$$

$$
\begin{aligned}
& T_{1}=\varepsilon_{i j}^{-1} B_{i} B_{i}^{t}+\varepsilon_{i j}^{-1} H_{b i} \Delta_{b i} \Delta_{b i}^{t} H_{b i}^{t}+\varepsilon_{i j}^{-1} P_{1} E_{a i}^{t} E_{a i} P_{1}+\varepsilon_{i j}^{-1} P_{1} K_{j}^{t} E_{b i}^{t} E_{b i} K_{j} P_{1} \\
& +\varepsilon_{i j} H_{a i} \Delta_{a i} \Delta_{a i}^{t} H_{a i}^{t}+\varepsilon_{i j} H_{b i} \Delta_{b i} \Delta_{b i}^{t} H_{b i}^{t}+\varepsilon_{i j}^{-1} P_{1} E_{a i}^{t} E_{a i} P_{1}+\varepsilon_{i j}^{-1} P_{1} K_{j}^{t} E_{b i}^{t} E_{b i} K_{j} P_{1}
\end{aligned}
$$


and

$$
\begin{aligned}
& T_{2}=\varepsilon_{i j} P_{2}^{-1} K_{j}^{t} K_{j} P_{2}^{-1}+\varepsilon_{i j} P_{2}^{-1} K_{j}^{t} E_{b i}^{t} E_{b i} K_{j} P_{2}^{-1}+\varepsilon_{i j} H_{a i} \Delta_{a i} \Delta_{a i}^{t} H_{a i}^{t} \\
& +\varepsilon_{i j} H_{b i} \Delta_{b i} \Delta_{b i}^{t} H_{b i}^{t}+\varepsilon_{i j} H_{b i} \Delta_{b i} \Delta_{b i}^{t} H_{b i}^{t}+\varepsilon_{i j}^{-1} P_{2}^{-1} K_{j}^{t} E_{b i}^{t} E_{b i} K_{j} P_{2}^{-1}
\end{aligned}
$$

From (15), (16), (17) and (21), we have:

$$
M_{D}(\bar{A}, X) \leq\left[\begin{array}{cc}
D_{1}+T_{1} & 0 \\
0 & D_{2}+T_{2}
\end{array}\right]=\left[\begin{array}{cc}
R_{1} & 0 \\
0 & R_{2}
\end{array}\right]
$$

In order to verify (14), we must have:

$$
\left[\begin{array}{cc}
R_{1} & 0 \\
0 & R_{2}
\end{array}\right]<0
$$

Which implies:

$$
\left\{\begin{array}{l}
R_{1}<0 \\
R_{2}<0
\end{array}\right.
$$

First, from (24), by using (3), using the Schur's complement (Boyd \& al, 1994) as well as the introduction of the new variable: $V_{i}=K_{j} P_{1}$ :

$$
\begin{aligned}
& R_{1}<0 \\
& \Leftrightarrow\left[\begin{array}{ccccc}
D_{i j} & P_{1} E_{a i}^{t} & V_{j}^{t} E_{b i}^{t} & B_{i} & H_{b i} \\
E_{a i} P_{1} & -0.5 \varepsilon_{i j} I & 0 & 0 & 0 \\
E_{b i} V_{j} & 0 & -0.5 \varepsilon_{i j} I & 0 & 0 \\
B_{i}^{t} & 0 & 0 & -\varepsilon_{i j} I & 0 \\
H_{b i}^{t} & 0 & 0 & 0 & -\varepsilon_{i j} I
\end{array}\right]<0
\end{aligned}
$$

Where $I$ is always the identity matrix of appropriate dimension and $D_{i j}=A_{i} P_{1}+P_{1} A_{i}^{t}+B_{i} V_{j}+V_{j}^{t} B_{i}^{t}+\varepsilon_{i j} H_{a i} H_{a i}^{t}+\varepsilon_{i j} H_{b i} H_{b i}^{t}$

Then, from (24), by using (3), using the Schur's complement (Boyd \& al, 1994) as well as the introduction of the new variable: $W_{i}=P_{2} G_{i}$ :

$$
\begin{aligned}
& R_{2}<0 \\
& \Leftrightarrow\left[\begin{array}{ccccc}
D_{i j}^{*} & K_{j}^{t} E_{b i}^{t} & P_{2} H_{a i} & P_{2} H_{b i} & K_{j}^{t} \\
E_{b i} K_{j} & -\varepsilon_{i j}^{-1} I & 0 & 0 & 0 \\
H_{a i}^{t} P_{2} & 0 & -\varepsilon_{i j}^{-1} I & 0 & 0 \\
H_{b i}^{t} P_{2} & 0 & 0 & -0.5 \varepsilon_{i j}^{-1} I & 0 \\
K_{j} & 0 & 0 & 0 & -\varepsilon_{i j}^{-1} I
\end{array}\right]<0
\end{aligned}
$$


Where $D_{i j}^{*}=P_{2} A_{i}+A_{i}^{t} P_{2}+W_{i} C_{j}+C_{j}^{t} W_{i}^{t}+\varepsilon_{i j}^{-1} K_{j}^{t} E_{b i}^{t} E_{b i} K_{j}$

Thus, conditions (12) and (13) yield for all $i, j$ from (25) and (26) and by using theorem 7 in (Tanaka \& al, 1998) which is necessary for LMI relaxations.

Remark 1: In lemma 1, the positive scalars $\varepsilon_{i j}$ are optimised unlike (Han \& al, 2000), (Lee \& al, 2001), (Tong \& Li, 2002), (Chadli \& El Hajjaji, 2006). We do not actually need to impose them to solve the set of LMIs. The conditions are thus less restrictive.

Remark 2: Note that it is a two-step procedure which allows us to design the controller and the observer separately. First, we solve (12) for decision variables $\left(P_{1}, K_{j}, \varepsilon_{i j}\right)$ and secondly, we solve (13) for decision variables $\left(P_{2}, G_{i}\right)$ by using the results from the first step. Furthermore, the controller and observer gains are given by: $G_{i}=P_{2}^{-1} W_{i}$ and $K_{j}=V_{j} P_{1}^{-1}$, respectively, for $i, j=1,2, \ldots, r$.

Remark 3: From lemma 1 and (10), the location of the poles associated with the state dynamics and with the estimation error dynamics is unknown. However, since the design algorithm is a two-step procedure, we can impose two pole placements separately, the first one for the state and the second one for the estimation error. In the following, we focus in the robust pole placement.

We hereafter give sufficient conditions to ensure the desired pole placements by using the LMI conditions of (Chilali \& Gahinet (1996) and (Chilali \& al, 1999) to the case of uncertain T-S fuzzy systems with unavailable state variables. Let us recall the definition of an LMI region and pole placement LMI constraints.

Definition 1 (Boyd \& al, 1994): A subset $D$ of the complex plane is called an LMI region if there exists a symmetric matrix $\alpha=\left[\alpha_{k l}\right] \in \mathfrak{R}^{m \times m}$ and a matrix $\beta=\left[\beta_{k l}\right] \in \mathfrak{R}^{m \times m}$ such as:

$$
D=\left\{z \in C: f_{D}(z)=\alpha+\beta z+\beta^{t} \bar{z}<0\right\}
$$

Definition 2 (Chilali and Gahinet, 1996): Let $D$ be a subregion of the left-half plane. A dynamical system described by: $\dot{x}=A x$ is called $D$-stable if all its poles lie in $D$. By extension, $\mathrm{A}$ is then called $D$-stable.

From the two previous definitions, the following theorem is given.

Theorem 1 (Chilali and Gahinet, 1996): Matrix $A$ is $D$-stable if and only if there exists a symmetric matrix $X>0$ such as

$$
M_{D}(A, X)=\alpha \otimes X+\beta \otimes A X+\beta^{t} \otimes X A^{t}<0
$$

where $\otimes$ denotes the Kronecker product.

From (10) and (11), let us define: $T_{i j}=\left(A_{i}+\Delta A_{i}\right)+\left(B_{i}+\Delta B_{i}\right) K_{j}$ and $S_{i j}=A_{i}+G_{i} C_{j}-\Delta B_{i} K_{j}$.

We hereafter give sufficient conditions to guarantee that $\sum_{i=1}^{r} \sum_{j=1}^{r} h_{i}(z(t)) h_{j}(z(t)) T_{i j}$ and $\sum_{i=1}^{r} \sum_{j=1}^{r} h_{i}(z(t)) h_{j}(z(t)) S_{i j}$ are $D_{T}$-stable and $D_{S}$-stable respectively in order to impose the dynamics of the state and the dynamics of the estimation error.

Lemma 2: Matrix $\sum_{i=1}^{r} \sum_{j=1}^{r} h_{i}(z(t)) h_{j}(z(t)) T_{i j}$ is $D_{T}$-stable if and only if there exist a symmetric matrix $P_{1}>0$ and positive scalars $\mu_{i j} \succ 0$ such as 


$$
\begin{aligned}
& \Omega_{i i} \leq 0, i=1, \ldots, r, \\
& \Omega_{i j}+\Omega_{j i} \leq 0, i<j \leq r .
\end{aligned}
$$

With

$$
\begin{aligned}
\Omega_{i j} & =\left(\begin{array}{ccc}
E_{i j} & \left(\beta^{t} \otimes P_{1} E_{a i}^{t}\right) & \left(\beta^{t} \otimes V_{j} E_{b i}^{t}\right) \\
\left(\beta \otimes E_{a i} P_{1}\right) & -\mu_{i j} I & 0 \\
\left(\beta \otimes E_{b i} V_{j}\right) & 0 & -\mu_{i j} I
\end{array}\right) \\
E_{i j} & =\xi_{i j}+\mu_{i j}\left(I \otimes H_{a i} H_{a i}^{t}\right)+\mu_{i j}\left(I \otimes H_{b i} H_{b i}^{t}\right) \\
\xi_{i j} & =\alpha \otimes P_{1}+\beta \otimes A_{i} P_{1}+\beta^{t} \otimes P_{1} A_{i}^{t}+\beta \otimes B_{i} V_{j}+\beta^{t} \otimes V_{j}^{t} B_{i}^{t} \\
V_{j} & =K_{j} P_{1}
\end{aligned}
$$

Proof: Using theorem 1, matrix $T_{i j}$ is $D_{T}$-stable if and only if there exists a symmetric matrix $X>0$ such that:

$$
M_{D_{T}}\left(T_{i j}, X\right)=\alpha \otimes X+\beta \otimes T_{i j} X+\beta^{t} \otimes X T_{i j}{ }^{t}<0
$$

$$
\begin{aligned}
& M_{D_{T}}\left(T_{i j}, X\right)=\alpha \otimes X+\beta \otimes A_{i} X+\beta^{t} \otimes X A_{i}^{t}+\beta \otimes B_{i} K_{j} X+\beta^{t} \otimes X K_{j}^{t} B_{i}^{t}+\beta \otimes H_{a i} \Delta_{a i} E_{a i} X \\
& +\beta^{t} \otimes X E_{a i}^{t} \Delta_{a i}^{t} H_{a i}^{t}+\beta \otimes H_{b i} \Delta_{b i} E_{b i} K_{j} X+\beta^{t} \otimes X K_{j}^{t} E_{b i}^{t} \Delta_{b i}^{t} H_{b i}^{t}
\end{aligned}
$$

Let $X=P_{1}$ and $V_{j}=K_{j} P_{1}$ :

$$
\begin{aligned}
M_{D_{T}}\left(T_{i j}, X\right) & =\xi_{i j}+\left(I \otimes H_{a i} \Delta_{a i}\right)\left(\beta \otimes E_{a i} P_{1}\right)+\left(\beta^{t} \otimes P_{1} E_{a i}^{t}\right)\left(I \otimes \Delta_{a i}^{t} H_{a i}^{t}\right)+\left(I \otimes H_{b i} \Delta_{b i}\right)\left(\beta \otimes E_{b i} V_{j}\right) \\
& +\left(\beta^{t} \otimes V_{j}^{t} E_{b i}^{t}\right)\left(I \otimes \Delta_{b i}^{t} H_{b i}^{t}\right)
\end{aligned}
$$

where

$$
\xi_{i j}=\alpha \otimes P_{1}+\beta \otimes A_{i} P_{1}+\beta^{t} \otimes P_{1} A_{i}^{t}+\beta \otimes B_{i} V_{j}+\beta^{t} \otimes V_{j}^{t} B_{i}^{t}
$$

Using the separation lemma (Shi \& al, 1992) and (3), we obtain:

$$
\begin{aligned}
& M_{D_{T}}\left(T_{i j}, X\right) \leq \xi_{i j}+\mu_{i j}\left(I \otimes H_{a i} H_{a i}^{t}\right)+\mu_{i j}^{-1}\left(\beta^{t} \otimes P_{1} E_{a i}^{t}\right)\left(\beta \otimes E_{a i} P_{1}\right) \\
& +\mu_{i j}\left(I \otimes H_{b i} H_{b i}^{t}\right)+\mu_{i j}^{-1}\left(\beta^{t} \otimes V_{j}^{t} E_{b i}^{t}\right)\left(\beta \otimes E_{b i} V_{j}\right)
\end{aligned}
$$

Thus, matrix $T_{i j}$ is $D_{T}$-stable if:

$$
\begin{aligned}
& \xi_{i j}+\mu_{i j}\left(I \otimes H_{a i} H_{a i}^{t}\right)+\mu_{i j}\left(I \otimes H_{b i} H_{b i}^{t}\right)+\mu_{i j}^{-1}\left(\beta^{t} \otimes P_{1} E_{a i}^{t}\right)\left(\beta \otimes E_{a i} P_{1}\right) \\
& +\mu_{i j}^{-1}\left(\beta^{t} \otimes V_{j}^{t} E_{b i}^{t}\right)\left(\beta \otimes E_{b i} V_{j}\right) \prec 0
\end{aligned}
$$

Where, of course, $\mu_{i j} \in \Re \forall i, j$ 
By using the Schur's complement (Boyd \& al, 1994),

$$
\begin{aligned}
& \left(\begin{array}{ccc}
E_{i j} & \left(\beta^{t} \otimes P_{1} E_{a i}^{t}\right) & \left(\beta^{t} \otimes V_{j} E_{b i}^{t}\right) \\
\left(\beta \otimes E_{a i} P_{1}\right) & -\mu_{i j} I & 0 \\
\left(\beta \otimes E_{b i} V_{j}\right) & 0 & -\mu_{i j} I
\end{array}\right) \prec 0, \\
& E_{i j}=\xi_{i j}+\mu_{i j}\left(I \otimes H_{a i} H_{a i}^{t}\right)+\mu_{i j}\left(I \otimes H_{b i} H_{b i}^{t}\right) .
\end{aligned}
$$

Thus, conditions (29) easily yield for all $i, j$.

Lemma 3: Matrix $\sum_{i=1}^{r} \sum_{j=1}^{r} h_{i}(z(t)) h_{j}(z(t)) S_{i j}$ is $D_{S^{-}}$-stable if and only if there exist a symmetric matrix $P_{2}>0$, matrices $W_{i}, K_{j}$ and positive scalars $\lambda_{i j} \succ 0$ such as

with

$$
\begin{aligned}
& \Phi_{i i} \leq 0, \quad i=1, \ldots, r \\
& \Phi_{i j}+\Phi_{j i} \leq 0, i<j \leq r
\end{aligned}
$$

$$
\begin{aligned}
& \Phi_{i j}=\left(\begin{array}{cc}
R_{i j}+\lambda_{i j}\left(\beta^{t} \otimes K_{j}^{t} E_{b i}^{t}\right)\left(\beta \otimes E_{b i} K_{j}\right) & I \otimes P_{2} H_{b i} \\
I \otimes H_{b i}^{t} P_{2} & -\lambda_{i j} I
\end{array}\right) \\
& R_{i j}=\alpha \otimes P_{2}+\beta \otimes P_{2} A_{i}+\beta^{t} \otimes A_{i}^{t} P_{2}+\beta \otimes W_{i} C_{j}+\beta^{t} \otimes C_{j}^{t} W_{i}^{t} \\
& W_{i}=P_{2} G_{i}
\end{aligned}
$$

Proof: Same lines as previously can be used to prove this lemma.

Let:

$$
\begin{aligned}
& M_{D_{S}}\left(S_{i j}, X\right)=\alpha \otimes X+\beta \otimes A_{i} X+\beta^{t} \otimes X A_{i}^{t}+\beta \otimes G_{i} C_{j} X+\beta^{t} \otimes X C_{j}^{t} G_{i}^{t} \\
& -\beta^{t} \otimes X K_{j}^{t} E_{b i}^{t}\left(I \otimes \Delta_{b i}^{t} H_{b i}^{t}\right)-\left(I \otimes \Delta_{b i} H_{b i}\right)\left(\beta \otimes E_{b i} K_{j} X\right)<0
\end{aligned}
$$

Using the separation lemma (Shi \& al, 1992), by pre- and post- multiplying by $I \otimes X^{-1}$, we obtain:

$$
\begin{aligned}
& \alpha \otimes X^{-1}+\beta \otimes\left(X^{-1} A_{i}\right)+\beta^{t} \otimes\left(A_{i}^{t} X^{-1}\right)+\beta \otimes\left(X^{-1} G_{i} C_{j}\right)+\beta^{t} \otimes\left(C_{j}^{t} G_{i}^{t} X^{-1}\right) \\
& +\lambda_{i j}\left(\beta^{t} \otimes K_{j}^{t} E_{b i}^{t}\right)\left(\beta \otimes E_{b i} K_{j}\right)+1 / \lambda_{i j}\left(I \otimes X^{-1} H_{b i}\right)\left(I \otimes H_{b i}^{t} X^{-1}\right)<0
\end{aligned}
$$

Where, of course, $\lambda_{i j} \in \mathfrak{R} \forall i, j$

Thus, by using the Schur's complement (Boyd \& al, 1994) as well as by defining $P_{2}=X^{-1}$ :

$$
\Phi_{i j}=\left(\begin{array}{cc}
\alpha \otimes P_{2}+\beta \otimes P_{2} A_{i}+\beta^{t} \otimes A_{i}^{t} P_{2}+\beta \otimes P_{2} G_{i} C_{j}+\beta^{t} \otimes C_{j}^{t} G_{i}^{t} P_{2}+\lambda_{i j}\left(\beta^{t} \otimes K_{j}^{t} E_{b i}^{t}\right)\left(\beta \otimes E_{b i} K_{j}\right) & I \otimes P_{2} H_{b i} \\
I \otimes H_{b i}^{t} P_{2} & -\lambda_{i j} I
\end{array}\right)<0
$$

By using $W_{i}=X^{-1} G_{i}$, conditions (38) easily yield for all $i, j$. The lemma proof is given. 
Remark 4: Any kind of LMI region (disk, vertical strip, conic sector) may be easily used for $D_{S}$ and $D_{T}$.

From lemma 2 and lemma 3, we have imposed the dynamics of the state as well as the dynamics of the estimation error. But from (10), the estimation error dynamics depend on the state. If the state dynamics are slow, we will have a slow convergence of the estimation error to the equilibrium point zero in spite of its own fast dynamics. So in this paper, we add an algorithm using the $H_{\infty}$ approach to ensure that the estimation error converges faster to the equilibrium point zero.

We know from (10) that:

$$
\begin{aligned}
& \dot{e}(t)=\sum_{i=1}^{r} \sum_{j=1}^{r} h_{i}(z(t)) h_{j}(z(t))\left(A_{i}+G_{i} C_{j}-\Delta B_{i} K_{j}\right) e(t) \\
& +\sum_{i=1}^{r} \sum_{j=1}^{r} h_{i}(z(t)) h_{j}(z(t)) S_{i j}\left(\Delta A_{i}+\Delta B_{i} K_{j}\right) x(t)
\end{aligned}
$$

This equation is equivalent to the following system:

$$
\left[\begin{array}{c}
\dot{e} \\
e
\end{array}\right]=\sum_{i=1}^{r} \sum_{j=1}^{r} h_{i}(z(t)) h_{j}(z(t))\left(\left[\begin{array}{cc}
A_{i}+G_{i} C_{j}-\Delta B_{i} K_{j} & \Delta A_{i}+\Delta B_{i} K_{j} \\
I & 0
\end{array}\right]\left[\begin{array}{l}
e \\
x
\end{array}\right]\right)
$$

The objective is to minimize the $L_{2}$ gain from $x(t)$ to $e(t)$ in order to guarantee that the error between the state and its estimation converges faster to zero. Thus, we define the following $H_{\infty}$ performance criterion under zero initial conditions:

$$
\int_{0}^{\infty}\left\{e^{t}(t) e(t)-\gamma^{2} x^{t}(t) x(t)\right\} d t<0
$$

where $\gamma \in \mathfrak{R}^{+^{*}}$ has to be minimized. Note that the signal $x(t)$ is square integrable because of lemma 1.

We give the following lemma to satisfy the $H_{\infty}$ performance.

Lemma 4: If there exist symmetric positive definite matrix $P_{2}$, matrices $W_{i}$ and positive scalars $\gamma \succ 0, \beta_{i j} \succ 0$ such as

With

$$
\begin{aligned}
& \Gamma_{i i} \leq 0, i=1, \ldots, r \\
& \Gamma_{i j}+\Gamma_{j i} \leq 0, i<j \leq r
\end{aligned}
$$

$$
\Gamma_{i j}=\left[\begin{array}{cccc}
Z_{i j} & P_{2} H_{b i} & P_{2} H_{a i} & -\beta_{i j} K_{j}^{t} E_{b i}^{t} E_{b i} K_{j} \\
H_{b i}^{t} P_{2} & -\beta_{i j} I & 0 & 0 \\
H_{a i}^{t} P_{2} & 0 & -\beta_{i j} I & 0 \\
-\beta_{i j} K_{j}^{t} E_{b i}^{t} E_{b i} K_{j} & 0 & 0 & U_{i j}
\end{array}\right]
$$




$$
\begin{gathered}
Z_{i j}=P_{2} A_{i}+A_{i}^{t} P_{2}+W_{i} C_{j}+C_{j}^{t} W_{i}^{t}+I+\beta_{i j} K_{j}^{t} E_{b i}^{t} E_{b i} K_{j} \\
U_{i j}=-\gamma^{2} I+\beta_{i j} K_{j}^{t} E_{b i}^{t} E_{b i} K_{j}+\beta_{i j} E_{a i}^{t} E_{a i}
\end{gathered}
$$

Then, the dynamic system:

$$
\left[\begin{array}{c}
\dot{e} \\
e
\end{array}\right]=\sum_{i=1}^{r} \sum_{j=1}^{r} h_{i}(z(t)) h_{j}(z(t))\left[\begin{array}{cc}
A_{i}+G_{i} C_{j}-\Delta B_{i} K_{j} & \Delta A_{i}+\Delta B_{i} K_{j} \\
I & 0
\end{array}\right]\left[\begin{array}{l}
e \\
x
\end{array}\right]
$$

satisfies the $H_{\infty}$ performance with a $L_{2}$ gain equal or less than $\gamma(44)$.

Proof: Applying the bounded real lemma (Boyd \& al, 1994), the system described by the following dynamics:

$$
\dot{e}(t)=\left(A_{i}+G_{i} C_{j}-\Delta B_{i} K_{j}\right) e(t)+\left(\Delta A_{i}+\Delta B_{i} K_{j}\right) x(t)
$$

satisfies the $H_{\infty}$ performance corresponding to the $L_{2}$ gain $\gamma$ performance if and only if there exists $P_{2}=P_{2}^{T}>0$ :

$$
\begin{aligned}
& \left(A_{i}+G_{i} C_{j}-\Delta B_{i} K_{j}\right)^{t} P_{2}+P_{2}\left(A_{i}+G_{i} C_{j}-\Delta B_{i} K_{j}\right) \\
& +P_{2}\left(\Delta A_{i}+\Delta B_{i} K_{j}\right)\left(\gamma^{2} I\right)^{-1}\left(\Delta A_{i}+\Delta B_{i} K_{j}\right)^{t} P_{2}+I \prec 0
\end{aligned}
$$

Using the Schur's complement, (Boyd \& al, 1994) yields

$$
\underbrace{\left[\begin{array}{cc}
J_{i j} & P_{2} \Delta A_{i}+P_{2} \Delta B_{i} K_{j} \\
\Delta A_{i}^{t} P_{2}+K_{j}^{t} \Delta B_{i}^{t} P_{2} & -\gamma^{2} I
\end{array}\right]}_{\Theta_{i j}} \prec 0
$$

where

$$
J_{i j}=P_{2} A_{i}+A_{i}^{t} P_{2}+P_{2} G_{i} C_{j}+C_{j}^{t} G_{i}^{t} P_{2}-P_{2} \Delta B_{i} K_{j}-K_{j}^{t} \Delta B_{i}^{t} P_{2}+I
$$

We get:

$$
\Theta_{i j}=\left[\begin{array}{cc}
P_{2} A_{i}+A_{i}^{t} P_{2}+P_{2} G_{i} C_{j}+C_{j}^{t} G_{i}^{t} P_{2}+I & 0 \\
0 & -\gamma^{2} I
\end{array}\right]+\underbrace{\left[\begin{array}{cc}
-P_{2} \Delta B_{i} K_{j}-K_{j}^{t} \Delta B_{i}^{t} P_{2} & P_{2} \Delta A_{i}+P_{2} \Delta B_{i} K_{j} \\
\Delta A_{i}^{t} P_{2}+K_{j}^{t} \Delta B_{i}^{t} P_{2} & 0
\end{array}\right]}_{\Delta_{i j}}
$$

By using the separation lemma (Shi \& al, 1992) yields

$$
\Delta_{i j} \leq \beta_{i j}\left[\begin{array}{cc}
K_{j}^{t} E_{b i}^{t} E_{b i} K_{j} & -K_{j}^{t} E_{b i}^{t} E_{b i} K_{j} \\
-K_{j}^{t} E_{b i}^{t} E_{b i} K_{j} & K_{j}^{t} E_{b i}^{t} E_{b i} K_{j}+E_{a i}^{t} E_{a i}
\end{array}\right]+\beta_{i j}^{-1}\left[\begin{array}{cc}
P_{2} H_{b i} \Delta_{b i} \Delta_{b i}^{t} H_{b i}^{t} P_{2}+P_{2} H_{a i} \Delta_{a i} \Delta_{a i}^{t} H_{a i}^{t} P_{2} & 0 \\
0 & 0
\end{array}\right]
$$

With substitution into $\Theta_{i j}$ and defining a variable change: $W_{i}=P_{2} G_{i}$, yields 


$$
\Theta_{i j} \leq\left[\begin{array}{cc}
Q_{i j} & -\beta_{i j} K_{j}^{t} E_{b i}^{t} E_{b i} K_{j} \\
-\beta_{i j} K_{j}^{t} E_{b i}^{t} E_{b i} K_{j} & -\gamma^{2} I+\beta_{i j} K_{j}^{t} E_{b i}^{t} E_{b i} K_{j}+\beta_{i j} E_{a i}^{t} E_{a i}
\end{array}\right]
$$

where

$$
\begin{aligned}
& \mathrm{Q}_{\mathrm{ij}}=\mathrm{R}_{\mathrm{ij}}+\beta_{\mathrm{ij}}^{-1} \mathrm{P}_{2} \mathrm{H}_{\mathrm{bi}} \Delta_{\mathrm{bi}} \Delta_{\mathrm{bi}}^{\mathrm{t}} \mathrm{H}_{\mathrm{bi}}^{\mathrm{t}} \mathrm{P}_{2}+\varepsilon_{\mathrm{ij}}^{-1} \mathrm{P}_{2} \mathrm{H}_{\mathrm{ai}} \Delta_{\mathrm{ai}} \Delta_{\mathrm{ai}}^{\mathrm{t}} \mathrm{H}_{\mathrm{ai}}^{\mathrm{t}} \mathrm{P}_{2}, \\
& \mathrm{R}_{\mathrm{ij}}=\mathrm{P}_{2} \mathrm{~A}_{\mathrm{i}}+\mathrm{A}_{\mathrm{i}}^{\mathrm{t}} \mathrm{P}_{2}+\mathrm{W}_{\mathrm{i}} \mathrm{C}_{\mathrm{j}}+\mathrm{C}_{\mathrm{j}}^{\mathrm{t}} \mathrm{W}_{\mathrm{i}}^{\mathrm{t}}+\mathrm{I}+\beta_{\mathrm{ij}} \mathrm{K}_{\mathrm{j}}^{\mathrm{t}} \mathrm{E}_{\mathrm{bi}}^{\mathrm{t}} \mathrm{E}_{\mathrm{bi}} \mathrm{K}_{\mathrm{j}} .
\end{aligned}
$$

Thus, from the following condition

$$
\left[\begin{array}{cc}
Q_{i j} & -\beta_{i j} K_{j}^{t} E_{b i}^{t} E_{b i} K_{j} \\
-\beta_{i j} K_{j}^{t} E_{b i}^{t} E_{b i} K_{j} & -\gamma^{2} I+\beta_{i j} K_{j}^{t} E_{b i}^{t} E_{b i} K_{j}+\beta_{i j} E_{a i}^{t} E_{a i}
\end{array}\right] \prec 0
$$

and using the Schur's complement (Boyd \& al, 1994), theorem 7 in ( Tanaka \& al, 1998) and (3), condition (46) yields for all $i, j$.

Remark 5: In order to improve the estimation error convergence, we obtain the following convex optimization problem: minimization $\gamma$ under the LMI constraints (46).

From lemma 1, 2, 3 and 4 yields the following theorem:

Theorem 2: The closed-loop uncertain fuzzy system (10) is robustly stabilizable via the observer-based controller (8) with control performances defined by a pole placement constraint in LMI region $D_{T}$ for the state dynamics, a pole placement constraint in LMI region $D_{S}$ for the estimation error dynamics and a $L_{2}$ gain $\gamma$ performance (45) as small as possible if first, LMI systems (12) and (29) are solvable for the decision variables $\left(P_{1}, K_{j}, \varepsilon_{i j}, \mu_{i j}\right)$ and secondly, LMI systems (13), (38), (46) are solvable for the decision variables $\left(P_{2}, G_{i}, \lambda_{i j}, \beta_{i j}\right)$. Furthermore, the controller and observer gains are $K_{j}=V_{j} P_{1}^{-1}$ and $G_{i}=P_{2}^{-1} W_{i}$, respectively, for $i, j=1,2, \ldots, r$.

Remark 6: Because of uncertainties, we could not use the separation property but we have overcome this problem by designing the fuzzy controller and observer in two steps with two pole placements and by using the $H_{\infty}$ approach to ensure that the estimation error converges faster to zero although its dynamics depend on the state.

Remark 7: Theorem 2 also proposes a two-step procedure: the first step concerns the fuzzy controller design by imposing a pole placement constraint for the poles linked to the state dynamics and the second step concerns the fuzzy observer design by imposing the second pole placement constraint for the poles linked to the error estimation dynamics and by minimizing the $H_{\infty}$ performance criterion (18). The designs of the observer and the controller are separate but not independent.

\section{Numerical example}

In this section, to illustrate the validity of the suggested theoretical development, we apply the previous control algorithm to the following academic nonlinear system (Lauber, 2003): 


$$
\left\{\begin{aligned}
\dot{x}_{1}(t)= & \left(\cos ^{2}\left(x_{2}(t)\right)-\frac{1}{1+x_{1}^{2}(t)}\right) x_{2}(t)+\left(1+\frac{1}{1+x_{1}^{2}(t)}\right) u(t) \\
\dot{x}_{2}(t)= & b\left(1+\frac{1}{1+x_{1}^{2}(t)}\right) \sin \left(x_{2}(t)\right)-1.5 x_{1}(t)-3 x_{2}(t) \\
& +\left(a \cos ^{2}\left(x_{2}(t)\right)-2\right) u(t) \\
y(t)= & x_{1}(t)
\end{aligned}\right.
$$

$y \in \Re$ is the system output, $u \in \Re$ is the system input, $x=\left[\begin{array}{ll}x_{1} & x_{2}\end{array}\right]^{t}$ is the state vector which is supposed to be unmeasurable. What we want to find is the control law $u$ which globally stabilizes the closed-loop and forces the system output to converge to zero but by imposing a transient behaviour.

Since the state vector is supposed to be unmeasurable, an observer will be designed.

The idea here is thus to design a fuzzy observer-based robust controller from the nonlinear system (57). The first step is to obtain a fuzzy model with uncertainties from (57) while the second step is to design the fuzzy control law from theorem 2 by imposing pole placement constraints and by minimizing the $H \infty$ criterion (46). Let us recall that, thanks to the pole placements, the estimation error converges faster to the equilibrium point zero and we impose the transient behaviour of the system output.

\section{First step:}

The goal is here to obtain a fuzzy model from (57).

By decomposing the nonlinear term $\frac{1}{1+x_{1}^{2}(t)}$ and integring nonlinearities of $x_{2}(t)$ into incertainties, then (20) is represented by the following fuzzy model:

Fuzzy model rule 1:

$$
\text { If } x_{1}(t) \text { is } M_{1} \text { then }\left\{\begin{array}{c}
\dot{x}=\left(A_{1}+\Delta A_{1}\right) x+\left(B_{1}+\Delta B_{1}\right) u \\
y=C x
\end{array}\right.
$$

Fuzzy model rule 2:

$$
\text { If } x_{1}(t) \text { is } M_{2} \text { then }\left\{\begin{array}{c}
\dot{x}=\left(A_{2}+\Delta A_{2}\right) x+\left(B_{2}+\Delta B_{2}\right) u \\
y=C x
\end{array}\right.
$$

where

$$
\begin{aligned}
& A_{1}=\left(\begin{array}{cc}
0 & 0.5 \\
-1.5 & -3+\frac{1+m}{2} b
\end{array}\right), B_{1}=\left(\begin{array}{c}
1 \\
\frac{a}{2}-2
\end{array}\right) \quad A_{2}=\left(\begin{array}{cc}
0 & 0.5 \\
-1.5 & -3+(1+m) b
\end{array}\right), B_{2}=\left(\begin{array}{c}
2 \\
\frac{a}{2}-2
\end{array}\right), \\
& H_{a i}=\left(\begin{array}{cc}
0.1 & 0 \\
0 & 0.1
\end{array}\right), H_{b i}=\left(\begin{array}{l}
0 \\
1
\end{array}\right), E_{b 1}=E_{b 2}=0.5|a| \\
& E_{a 1}=\left(\begin{array}{cc}
0 & 0.5 \\
0 & \frac{1-m}{2}|b|
\end{array}\right), E_{a 2}=\left(\begin{array}{cc}
0 & 0.5 \\
0 & (1-m)|b|
\end{array}\right), C=\left(\begin{array}{ll}
1 & 0
\end{array}\right), \\
& m=-0.2172, b=-0.5, a=2 \text { and } i=1,2
\end{aligned}
$$




\section{Second step:}

The control design purpose of this example is to place both the poles linked to the state dynamics and to the estimation error dynamics in the vertical strip given by: $\left(\begin{array}{ll}\alpha_{1} & \alpha_{2}\end{array}\right)=\left(\begin{array}{ll}-1 & -6\end{array}\right)$. The choice of the same vertical strip is voluntary because we wish to compare results of simulations obtained with and without the $H_{\infty}$ approach, in order to show by simulation the effectiveness of our approach.

The initial values of states are chosen: $x(0)=\left[\begin{array}{ll}-0.2 & -0.1\end{array}\right]$ and $\hat{x}(0)=\left[\begin{array}{ll}0 & 0\end{array}\right]$.

By solving LMIs of theorem 2, we obtain the following controller and observer gain matrices respectively:

$$
\mathrm{K}_{1}=\left[\begin{array}{ll}
-1.95 & -0.17
\end{array}\right], \mathrm{K}_{2}=\left[\begin{array}{ll}
-1.36 & -0.08
\end{array}\right], \mathrm{G}_{1}=\left[\begin{array}{ll}
-7.75 & -80.80
\end{array}\right]^{\mathrm{t}}, \mathrm{G}_{2}=\left[\begin{array}{ll}
-7.79 & -82.27
\end{array}\right]^{\mathrm{t}}
$$

The obtained $H_{\infty}$ criterion after minimization is:

$$
\gamma=0.3974
$$

Tables 1 and 2 give some examples of both nominal and uncertain system closed-loop pole values respectively. All these poles are located in the desired regions. Note that the uncertainties must be taken into account since we wish to ensure a global pole placement. That means that the poles of (10) belong to the specific LMI region, whatever uncertainties (2), (3). From tables 1 and 2, we can see that the estimation error pole values obtained using the $H_{\infty}$ approach are more distant (farther on the left) than the ones without the $H_{\infty}$ approach.

\begin{tabular}{|l|l|l|l|l|}
\hline & \multicolumn{2}{|l|}{ With the $H_{\infty}$ approach } & \multicolumn{2}{l|}{ Without the $H_{\infty}$ approach } \\
\cline { 2 - 5 } & Pole 1 & Pole 2 & Pole 1 & Pole 2 \\
\hline$A_{1}+B_{1} K_{1}$ & -1.8348 & -3.1403 & -1.8348 & -3.1403 \\
\hline$A_{2}+B_{2} K_{2}$ & -2.8264 & -3.2172 & -2.8264 & -3.2172 \\
\hline$A_{1}+G_{1} C_{1}$ & $-5.47+5.99 \mathrm{i}$ & $-5.47-5.99 \mathrm{i}$ & $-3.47+3.75 \mathrm{i}$ & $-3.47-3.75 \mathrm{i}$ \\
\hline$A_{2}+G_{2} C_{2}$ & $-5.59+6.08 \mathrm{i}$ & $-5.59-6.08 \mathrm{i}$ & $-3.87+3.96 \mathrm{i}$ & $-3.87-3.96 \mathrm{i}$ \\
\hline
\end{tabular}

Table 1. Pole values (nominal case).

\begin{tabular}{|l|l|l|l|l|}
\hline & \multicolumn{2}{|l|}{ With the $H_{\infty}$ approach } & \multicolumn{2}{l|}{ Without the $H_{\infty}$ approach } \\
\hline & \multicolumn{1}{|l|}{ Pole 1} & Pole 2 & Pole 1 & Pole 2 \\
\hline$A_{1}+H_{a 1} E_{a 1}+\left(B_{1}+H_{b 1} E_{b 1}\right) K_{1}$ & $-2.56+.43 i$ & $-2.56-0.43 i$ & $-2.56+0.43 i$ & $-2.56-0.43 i$ \\
\hline$A_{2}+H_{a 2} E_{a 2}+\left(B_{2}+H_{b 2} E_{b 2}\right) K_{2}$ & $-3.03+0.70 i$ & $-3.032-0.70 i$ & $-3.03+0.70 i$ & $-3.03-0.70 i$ \\
\hline$A_{1}-H_{a 1} E_{a 1}+\left(B_{1}+H_{b 1} E_{b 1}\right) K_{1}$ & $-2.58+0.10 i$ & $-2.58-0.10 i$ & $-2.58+0.10 i$ & $-2.58-0.10 i$ \\
\hline$A_{2}-H_{a 2} E_{a 2}+\left(B_{2}+H_{b 2} E_{b 2}\right) K_{2}$ & $-3.09+0.54 i$ & $-3.09-0.54 i$ & $-3.09+0.54 i$ & $-3.09-0.54 i$ \\
\hline$A_{1}+G_{1} C_{1}-H_{b 1} E_{b 1} K_{1}$ & $-5.38+5.87 i$ & $-5.38-5.87 i$ & $-3.38+3.61 i$ & $-3.38-3.61 i$ \\
\hline$A_{2}+G_{2} C_{2}-H_{b 2} E_{b 2} K_{2}$ & $-5.55+6.01 i$ & $-5.55-6.01 i$ & $-3.83+3.86 i$ & $-3.83-3.86 i$ \\
\hline
\end{tabular}

Table 2. Pole values (extreme uncertain models). 
Figures 1 and 2 respectively show the behaviour of error $e_{1}(t)$ and $e_{2}(t)$ with and without the $H_{\infty}$ approach and also the behaviour obtained using only lemma 1 . We clearly see that the estimation error converges faster in the first case (with $H_{\infty}$ approach and pole placements) than in the second one (with pole placements only) as well as in the third case (without $H_{\infty}$ approach and pole placements). At last but not least, Figure 3 and 4 show respectively the behaviour of the state variables with and without the $H_{\infty}$ approach whereas Figure 5 shows the evolution of the control signal. From Figures 3 and 4 , we still have the same conclusion about the convergence of the estimation errors.

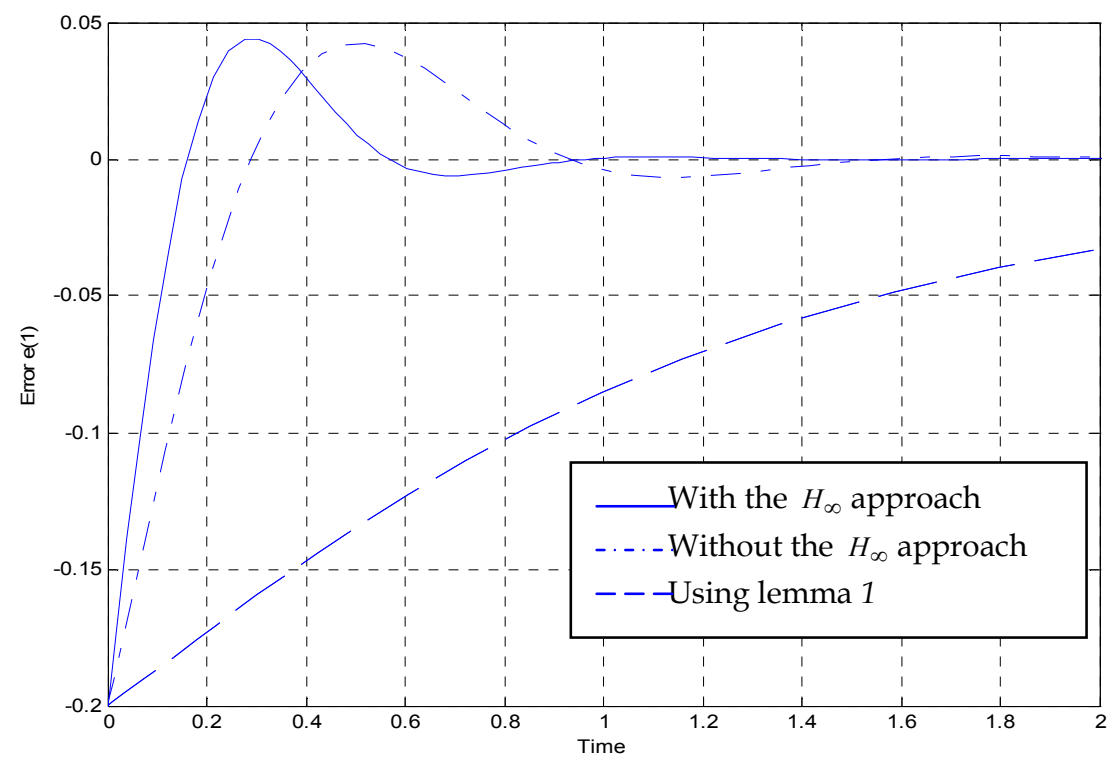

Fig. 1. Behaviour of error $e_{1}(t)$. 


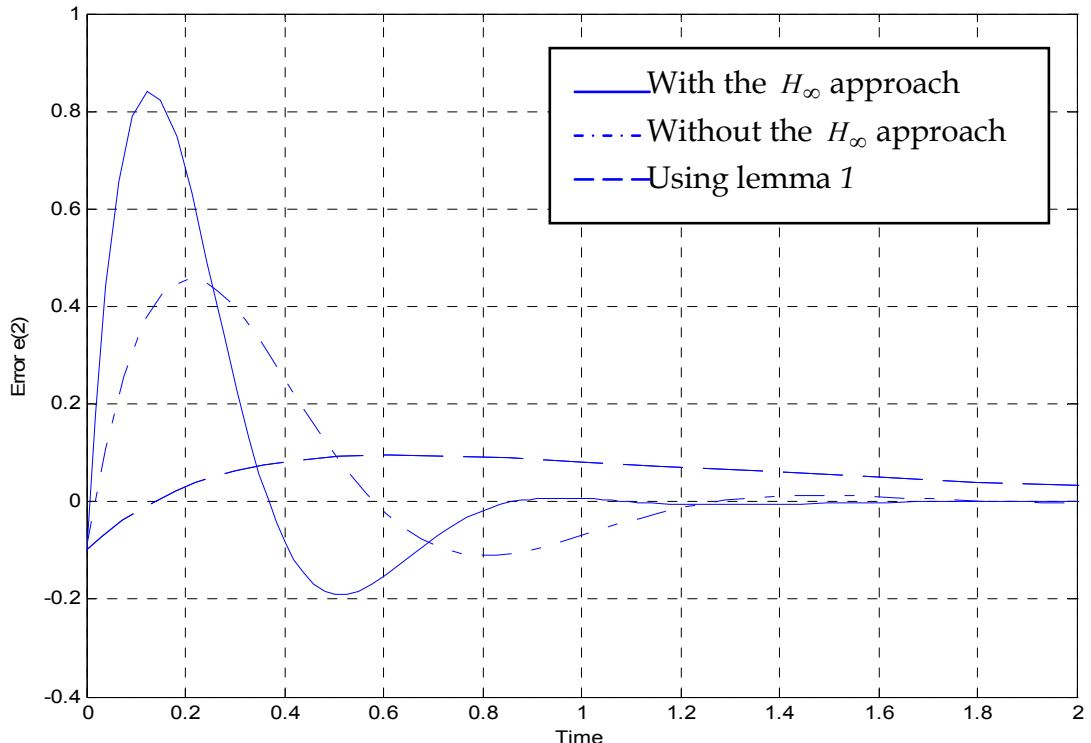

Fig. 2. Behaviour of error $e_{2}(t)$.
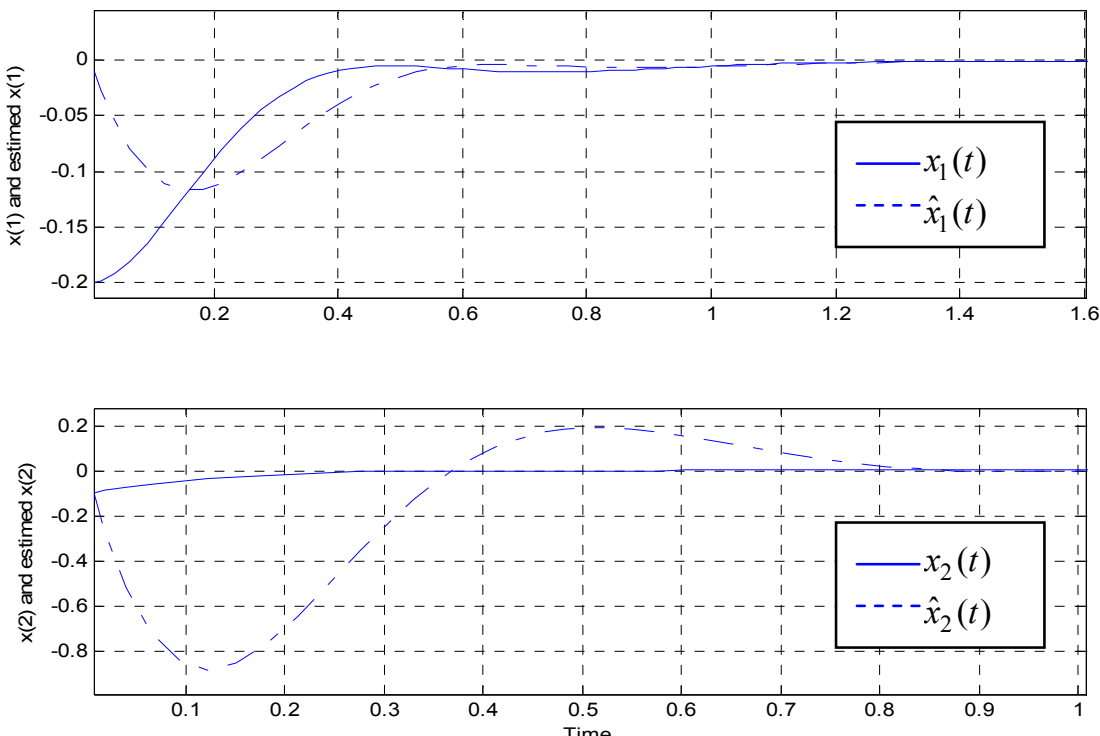

Fig. 3. Behaviour of the state vector and its estimation with the $H_{\infty}$ approach. 

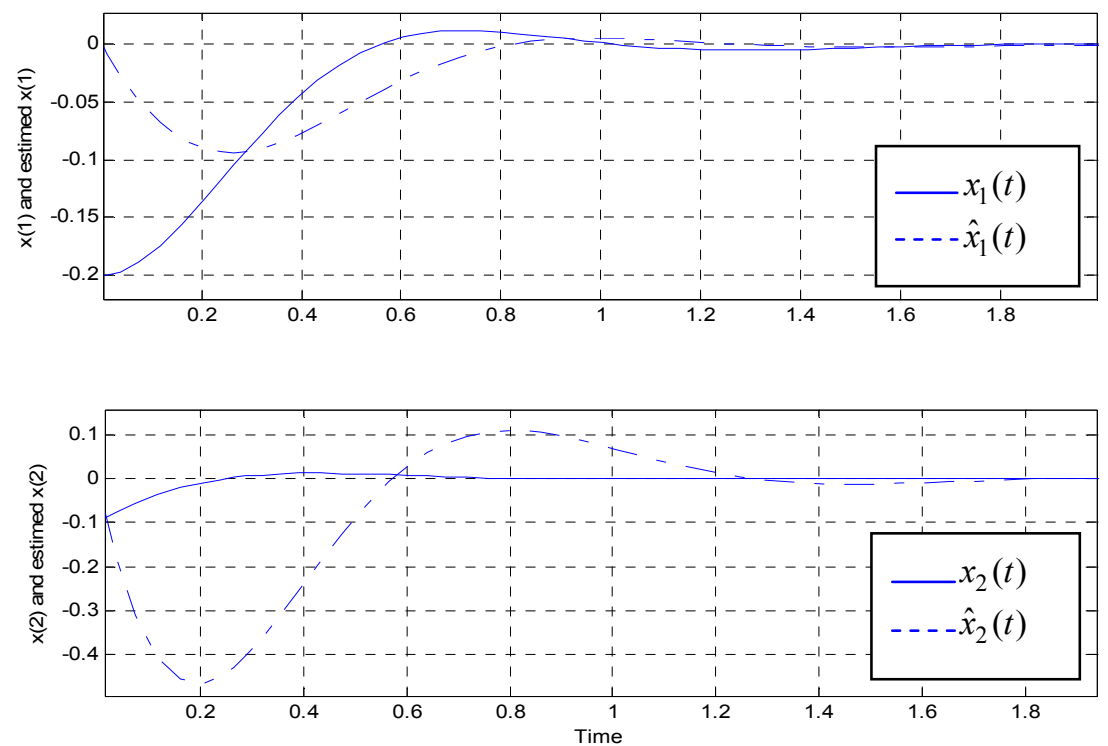

Fig. 4. Behaviour of the state and its estimation without the $H_{\infty}$ approach.

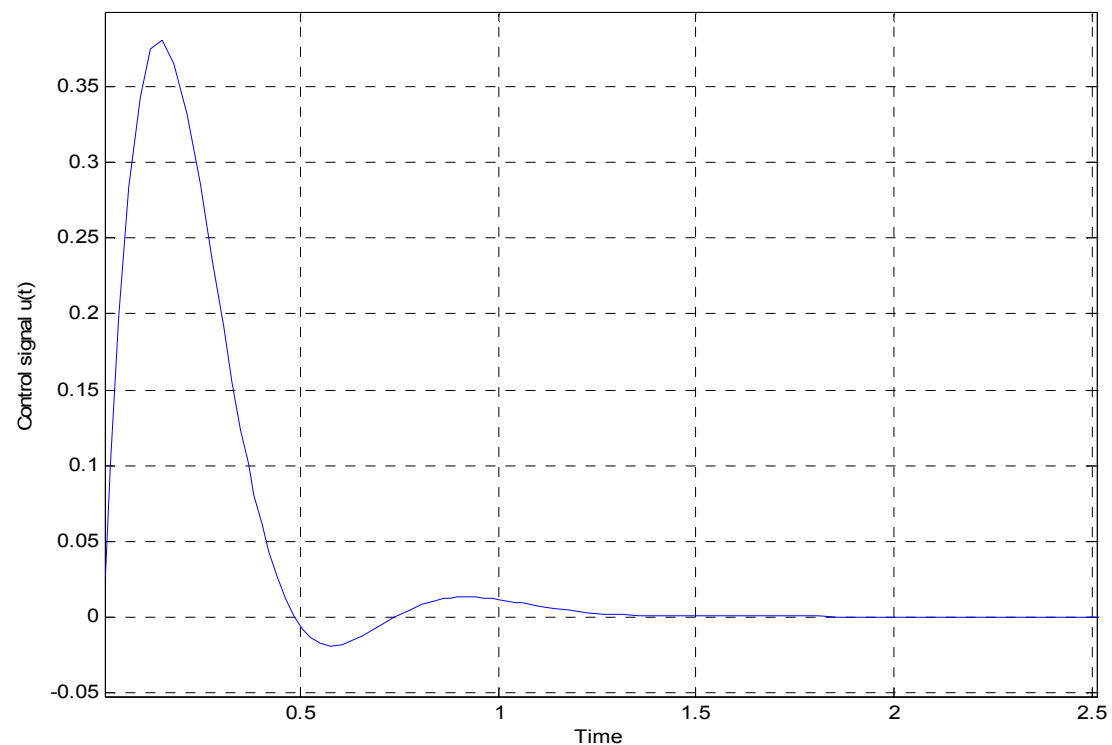

Fig. 5. Control signal evolution $u(t)$. 


\section{Conclusion}

In this chapter, we have developed robust pole placement constraints for continuous T-S fuzzy systems with unavailable state variables and with parametric structured uncertainties. The proposed approach has extended existing methods based on uncertain T-S fuzzy models. The proposed LMI constraints can globally asymptotically stabilize the closed-loop T-S fuzzy system subject to parametric uncertainties with the desired control performances. Because of uncertainties, the separation property is not applicable. To overcome this problem, we have proposed, for the design of the observer and the controller, a two-step procedure with two pole placements constraints and the minimization of a $\mathrm{H}_{\infty}$ performance criterion in order to guarantee that the estimation error converges faster to zero. Simulation results have verified and confirmed the effectiveness of our approach in controlling nonlinear systems with parametric uncertainties.

\section{References}

Chadli, M. \& El Hajjaji, A. (2006). Comment on observer-based robust fuzzy control of nonlinear systems with parametric uncertainties. Fuzzy Sets and Systems, Vol. 157, N॰9 (2006), pp. 1276-1281

Boyd, S.; El Ghaoui, L. \& Feron, E. \& Balkrishnan, V. (1994). Linear Matrix Inequalities in System and Control Theory, Society for Industrial and Applied Mathematics, SIAM, Philadelphia, USA

Chilali, M. \& Gahinet, P. (1996). $H_{\infty}$ Design with Pole Placement Constraints: An LMI Approach. IEEE Transactions on Automatic Control, Vol. 41, №3 (March 1996), pp. 358-367

Chilali, M.; Gahinet, P. \& Apkarian, P. (1999). Robust Pole Placement in LMI Regions. IEEE Transactions on Automatic Control, Vol. 44, N¹2 (December 1999), pp. 2257-2270

El Messoussi, W.; Pagès, O. \& El Hajjaji, A. (2005). Robust Pole Placement for Fuzzy Models with Parametric Uncertainties: An LMI Approach, Proceedings of the 4th Eusflat and $11^{\text {th }}$ LFA Congress, pp. 810-815, Barcelona, Spain, September, 2005

El Messoussi, W.; Pagès, O. \& El Hajjaji, A. (2006).Observer-Based Robust Control of Uncertain Fuzzy Dynamic Systems with Pole Placement Constraints: An LMI Approach, Proceedings of the IEEE American Control conference, pp. 2203-2208, Minneapolis, USA, June, 2006

Farinwata, S.; Filev, D. \& Langari, R. (2000). Fuzzy Control Synthesis and Analysis, John Wiley \& Sons, Ltd, pp. 267-282

Han, Z.X.; Feng, G. \& Walcott, B.L. \& Zhang, Y.M. (2000) . $H_{\infty}$ Controller Design of Fuzzy Dynamic Systems with Pole Placement Constraints, Proceedings of the IEEE American Control Conference, pp. 1939-1943, Chicago, USA, June, 2000

Hong, S. K. \& Nam, Y. (2003). Stable Fuzzy Control System Design with Pole Placement constraint: An LMI Approach. Computers in Industry, Vol. 51, Nº1 (May 2003), pp. 111

Kang, G.; Lee, W. \& Sugeno, M. (1998). Design of TSK Fuzzy Controller Based on TSK Fuzzy Model Using Pole Placement, Proceedings of the IEEE World Congress on Computational Intelligence, pp. 246 - 251, Vol. 1, N¹2, Anchorage, Alaska, USA, May, 1998 
Lauber J. (2003). Moteur à allumage commandé avec EGR: modélisation et commande non linéaires, $\mathrm{Ph}$. D. Thesis of the University of Valenciennes and Hainault-Cambresis, France, December 2003, pp. 87-88

Lee, H.J.; Park, J.B. \& Chen, G. (2001). Robust Fuzzy Control of Nonlinear Systems with Parametric Uncertainties. IEEE Transactions on Fuzzy Systems, Vol. 9, ํ2, (April 2001), pp. 369-379

Lo, J. C. \& Lin, M. L. (2004). Observer-Based Robust $H_{\infty}$ Control for Fuzzy Systems Using Two-Step Procedure. IEEE Transactions on Fuzzy Systems, Vol. 12, N³, (June 2004), pp. 350-359

Ma, X. J., Sun Z. Q. \& He, Y. Y. (1998). Analysis and Design of Fuzzy Controller and Fuzzy Observer. IEEE Transactions on Fuzzy Systems, Vol. 6, №1, (February 1998), pp. 4151

Shi, G., Zou Y. \& Yang, C. (1992). An algebraic approach to robust $H_{\infty}$ control via state feedback. System Control Letters, Vol. 18, N5 (1992), pp. 365-370

Tanaka, K.; Ikeda, T. \& Wang, H. O. (1998). Fuzzy Regulators and Fuzzy Observers: Relaxed Stability Conditions and LMI-Based Design. IEEE Transactions on Fuzzy Systems, Vol. 6, N², (May 1998), pp. 250-265

Tong, S. \& Li, H. H. (1995). Observer-based robust fuzzy control of nonlinear systems with parametric uncertainties. Fuzzy Sets and Systems, Vol. 131, N², (October 2002), pp. 165-184

Wang, S. G.; Shieh, L. S. \& Sunkel, J. W. (1995). Robust optimal pole-placement in a vertical strip and disturbance rejection in Structured Uncertain Systems. International Journal of System Science, Vol. 26, (1995), pp. 1839-1853

Wang, S. G.; Shieh, L. S. \& Sunkel, J. W. (1998). Observer-Based controller for Robust Pole Clustering in a vertical strip and disturbance rejection. International Journal of Robust and Nonlinear Control, Vol. 8, N5, (1998), pp. 1073-1084

Wang, S. G.; Yeh, Y. \& Roschke, P. N. (2001). Robust Control for Structural Systems with Parametric and Unstructured Uncertainties, Proceedings of the American Control Conference, pp. 1109-1114, Arlington, USA, June 2001

Xiaodong, L. \& Qingling, Z. (2003). New approaches to $H_{\infty}$ controller designs based on fuzzy observers for T-S fuzzy systems via LMI. Automatica, Vol. 39, N 9, (September 2003), pp. 1571-1582

Yoneyama, J; Nishikawa, M.; Katayama, H. \& Ichikawa, A. (2000). Output stabilization of Takagi-Sugeno fuzzy systems. Fuzzy Sets and Systems, Vol. 111, N², April 2000, pp. 253-266 


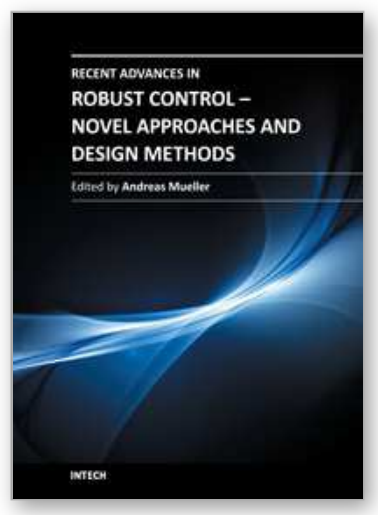

\author{
Recent Advances in Robust Control - Novel Approaches and \\ Design Methods \\ Edited by Dr. Andreas Mueller
}

ISBN 978-953-307-339-2

Hard cover, 462 pages

Publisher InTech

Published online 07, November, 2011

Published in print edition November, 2011

Robust control has been a topic of active research in the last three decades culminating in $\mathrm{H}$ 2/H_linfty and Imu design methods followed by research on parametric robustness, initially motivated by Kharitonov's theorem, the extension to non-linear time delay systems, and other more recent methods. The two volumes of Recent Advances in Robust Control give a selective overview of recent theoretical developments and present selected application examples. The volumes comprise 39 contributions covering various theoretical aspects as well as different application areas. The first volume covers selected problems in the theory of robust control and its application to robotic and electromechanical systems. The second volume is dedicated to special topics in robust control and problem specific solutions. Recent Advances in Robust Control will be a valuable reference for those interested in the recent theoretical advances and for researchers working in the broad field of robotics and mechatronics.

\title{
How to reference
}

In order to correctly reference this scholarly work, feel free to copy and paste the following:

Pagès Olivier and El Hajjaji Ahmed (2011). Observer-Based Robust Control of Uncertain Fuzzy Models with Pole Placement Constraints, Recent Advances in Robust Control - Novel Approaches and Design Methods, Dr. Andreas Mueller (Ed.), ISBN: 978-953-307-339-2, InTech, Available from:

http://www.intechopen.com/books/recent-advances-in-robust-control-novel-approaches-and-designmethods/observer-based-robust-control-of-uncertain-fuzzy-models-with-pole-placement-constraints

\section{INTECH}

open science | open minds

\section{InTech Europe}

University Campus STeP Ri

Slavka Krautzeka 83/A

51000 Rijeka, Croatia

Phone: +385 (51) 770447

Fax: +385 (51) 686166

www.intechopen.com

\section{InTech China}

Unit 405, Office Block, Hotel Equatorial Shanghai

No.65, Yan An Road (West), Shanghai, 200040, China

中国上海市延安西路65号上海国际贵都大饭店办公楼 405 单元

Phone: +86-21-62489820

Fax: $+86-21-62489821$ 
(C) 2011 The Author(s). Licensee IntechOpen. This is an open access article distributed under the terms of the Creative Commons Attribution 3.0 License, which permits unrestricted use, distribution, and reproduction in any medium, provided the original work is properly cited. 